\title{
The quantifier semigroup for bipartite graphs
}

\author{
Leonard J. Schulman* \\ Submitted: Jun 22, 2010; Accepted: May 17, 2011; Published: May 30, 2011 \\ Mathematics Subject Classification: 06A15, 06F05
}

\begin{abstract}
In a bipartite graph there are two widely encountered monotone mappings from subsets of one side of the graph to subsets of the other side: one corresponds to the quantifier "there exists a neighbor in the subset" and the other to the quantifier "all neighbors are in the subset." These mappings generate a partially ordered semigroup which we characterize in terms of "run-unimodal" words.
\end{abstract}

\section{Introduction}

Every bipartite graph $G \subseteq U \times V$ defines a Galois connection consisting of the following pair of maps between the boolean algebras $\{0,1\}^{U}$ and $\{0,1\}^{V}$ :

$$
\begin{array}{lll}
e:\{0,1\}^{U} \rightarrow\{0,1\}^{V} & \text { defined by } & e(S)=\{v: G(*, v) \cap S \neq \emptyset\} \\
a:\{0,1\}^{V} \rightarrow\{0,1\}^{U} & \text { defined by } & a(T)=\{u: G(u, *) \subseteq T\}
\end{array}
$$

Here $G(*, v)$ denotes $\{u:(u, v) \in G\}$, and $G(u, *)$ denotes $\{v:(u, v) \in G\}$. Thus, $e(S)$ consists of vertices $v$ for which there exists an edge connecting them to $S$; while $a(T)$ consists of vertices $u$ all of whose edges connect to $T$.

Each of the maps $e$ and $a$ is monotone increasing, and they satisfy the Galois identities

$$
e a e=e, \quad a e a=a .
$$

(These identities are equivalent to each other because of the identity $e \neg=\neg a$, where $\neg$ denotes complement with respect to $U$ or $V$ as appropriate.) Galois connections are a unifying framework for many closure operators on set systems, the closure being defined by either $e a$ or ae, depending on the situation $[8,2,9,11,10,1,13]$. Examples: (1) $U$ consists of the points of a topology, $V$ consists of its open sets, $G$ is membership. Here $a e S=$ the topological closure of $S$. (2) $U$ consists of the points of a Euclidean space, $V$

*schulman@caltech.edu. Caltech, Pasadena, CA 91125. Supported in part by NSF CCR-0326554, NSF CCF-0515342, NSA H98230-06-1-0074, and NSF CCF-0829909. 
consists of its closed affine halfspaces, $G$ is membership. Here aeS = the convex span of $S$. (3) $U$ consists of the points of a finite-dimensional vector space over some field, $V$ is the ring of polynomials in the coordinates, $G$ is the property of polynomial $v$ being nonzero at point $u$. Here $a e S$ is the variety spanning $S$, while $e a T$ is the complement of the radical of the ideal spanned by $V-T$.

The above pair of mappings also play a well-known role in the marriage theorem [3, $6,5,4]$ which states that the following are equivalent: (a) $G$ contains a matching of size $|U|$. (b) $|S| \leq|e S|$ for all $S \subseteq U$. (c) $|a T| \leq|T|$ for all $T \subseteq V$. But this will not be our focus.

Instead, we pursue the consequences of the fact that the mappings $e$ and $a$ make sense also in the reverse directions:

$$
\begin{array}{lll}
e:\{0,1\}^{V} \rightarrow\{0,1\}^{U} & \text { defined by } & e(T)=\{u: G(u, *) \cap T \neq \emptyset\} \\
a:\{0,1\}^{U} \rightarrow\{0,1\}^{V} & \text { defined by } & a(S)=\{v: G(*, v) \subseteq S\}
\end{array}
$$

With these definitions, any even-length word over the alphabet $\{a, e\}$ defines a monotone mapping from $\{0,1\}^{U}$ to itself. For instance, example (1) yields another familiar closure operation: $e a S=$ the topological interior of $S$.

Define the height function $h$ on words as follows: $h(e)=1 ; h(a)=-1$; the height of a word is the sum of the heights of its letters.

Definition 1. Let $F$ be the free semigroup of even-length words on $\{a, e\}$, and let $F^{0}$ be its sub-semigroup consisting of words of height 0 .

The relation $\leq$ is defined on $F$ by: $w \leq w^{\prime}$ if $w S \subseteq w^{\prime} S$ for all $G$ and all $S \subseteq U$.

The focus of this paper is the partial order on the following semigroup:

Definition 2. The quantifier semigroup for bipartite graphs, $\mathfrak{S}$, is defined to be the quotient semigroup of $F^{0}$ obtained by setting words $w$ and $w^{\prime}$ to be equal if $w \leq w^{\prime}$ and $w^{\prime} \leq w$. $\mathfrak{S}$ inherits $\leq$ as a partial order.

A first step to understanding the partial order on $\mathfrak{S}$ is the observation that (with 1 denoting the empty word),

$$
e a \leq 1 \leq a e
$$

The first inequality holds because, if $x \in U$ has a neighbor all of whose neighbors lie in

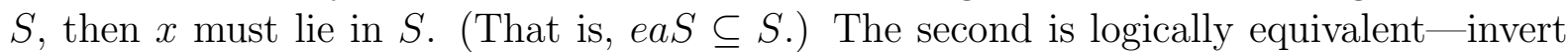
the partial order on the boolean algebra. (Or for a direct argument, note that if $x \in S$ then every neighbor of $x$ has a neighbor in $S$. That is, $S \subseteq$ aeS.) The pair of inequalities (1) imply the Galois identities $e a e=e$ and $a e a=a$, by

$$
e \leq e(a e)=(e a) e \leq e, \quad \text { and } \quad a \leq(a e) a=a(e a) \leq a
$$

As we shall see however, the Galois identities are only the first pair in a list of identities that characterize $\mathfrak{S}$. 


\section{The semigroup $\mathfrak{S}$}

Lemma 3. If every suffix of a word $v \in F^{0}$ is of nonnegative height then $v \geq 1$. If every suffix of $v$ is of nonpositive height, then $v \leq 1$.

Proof. The assertions are equivalent, so consider only the first. Argue by induction on the length of $v$. Parse $v=v_{2} a e v_{1}$ so that $e v_{1}$ is a suffix of greatest height. Apply ae $\geq 1$ to conclude $v \geq v_{2} 1 v_{1}=v_{2} v_{1} \geq 1$.

Corollary 4. $e^{n} a^{n} e^{n}=e^{n}$ and $a^{n} e^{n} a^{n}=a^{n}$ for any $n \geq 0$.

Proof. Again the assertions are equivalent and we consider only the first. Using Lemma 3: $e^{n} \geq\left(e^{n} a^{n}\right) e^{n}=e^{n}\left(a^{n} e^{n}\right) \geq e^{n}$.

Corollary 5. $\mathfrak{S}$ is idempotent.

Proof. Any $w \in F^{0}$ can be parsed into $w=w_{C} w_{B} w_{A}$ so that either $w_{A}$ is a suffix of greatest height and $w_{B} w_{A}$ is a suffix of least height, or else $w_{A}$ is a suffix of least height and $w_{B} w_{A}$ is a suffix of greatest height. Due to the symmetry of the cases we address only the former. Then $w_{B} w_{A} w_{C}$ is a word with nonnegative-height suffixes, and $w_{A} w_{C} w_{B}$ is a word with nonpositive-height suffixes. So

$w w=w_{C}\left(w_{B} w_{A} w_{C}\right) w_{B} w_{A} \geq w_{C} 1 w_{B} w_{A}=w=w_{C} w_{B} 1 w_{A} \geq w_{C} w_{B}\left(w_{A} w_{C} w_{B}\right) w_{A}=w w$.

Write a word $w$ as $w=a^{n_{s}} e^{n_{s-1}} \ldots a^{n_{2}} e^{n_{1}}$, with all exponents positive except possibly $n_{1}$ and $n_{s}$ which may be 0 . A key concept is that of a run-unimodal word:

Definition 6. $w$ is run-unimodal if $h(w)=0$ and if there does not exist a $j$ for which $n_{j}>0$ and $n_{j+1} \geq n_{j} \leq n_{j-1}$.

Equivalently, there exists an $r$ for which either $0 \leq n_{s}<n_{s-1}<\ldots<n_{r}>\ldots>n_{1} \geq$ 0 or $0 \leq n_{s}<n_{s-1}<\ldots<n_{r+1}=n_{r}>\ldots>n_{1} \geq 0$.

Let $\mathcal{G}$ denote the set of identities $\left\{e^{n} a^{n} e^{n}=e^{n}, a^{n} e^{n} a^{n}=a^{n}\right\}_{n \geq 1}$. Corollary 4 shows that $\mathfrak{S}$ is a quotient of $F^{0} / \mathcal{G}$.

Our main result is to show that there are no further relations in $\mathfrak{S}$, i.e.:

Theorem 7. $\mathfrak{S}=F^{0} / \mathcal{G}$.

So, for example, the words $e a^{2} e$ and $a e^{2} a$ are incomparable with each other and with the identity.

In preparation for the proof of this theorem we note that there is a nice representation for $F^{0} / \mathcal{G}$ :

Lemma 8. Every equivalence class in $F^{0} / \mathcal{G}$ has a unique shortest representative, and it is run-unimodal.

This lemma allows for a good visual representation of the elements of $F^{0} / \mathcal{G}$, which, thanks to Theorem 7, is actually a representation of $\mathfrak{S}$ itself. See Figure 1 for an illustration of small portions of $\mathfrak{S}$. 
Proof. If $w=a^{n_{s}} e^{n_{s-1}} \ldots a^{n_{2}} e^{n_{1}}$ is not run-unimodal, let $j$ be such that $n_{j}>0$ and $n_{j+1} \geq n_{j} \leq n_{j-1}$. Then $w$ is equivalent to the shorter word with the exponent sequence $n_{s}, \ldots, n_{j+2}, n_{j+1}-n_{j}+n_{j-1}, n_{j-2}, \ldots, n_{1}$.

We next show that different run-unimodal words are inequivalent in $F^{0} / \mathcal{G}$ by arguing that relations in $\mathcal{G}$ leave invariant four lists of numbers, which distinguish among rununimodal words: the lists of suffix peaks, suffix valleys, prefix peaks and prefix valleys.

These notions are defined later in the proof of this lemma, but it will be easier to follow that definition after first seeing what peaks and valleys are in the special case of run-unimodal words (with $r$ as following Definition 6):

The peaks occur at the "odd suffixes":

$$
h\left(e^{n_{s-1}} \ldots a^{n_{2}} e^{n_{1}}\right), \quad h\left(e^{n_{3}} a^{n_{2}} e^{n_{1}}\right), \quad \ldots, \quad h\left(e^{n_{1}}\right) .
$$

This list of numbers is unimodal. The suffix peaks are the increasing terms at the right of the sequence, while the prefix peaks are the decreasing terms at the left of the sequence. In case the sequence has a unique maximum, it is considered as both a suffix and prefix peak. Similarly, the valleys are

$$
h\left(a^{n_{s}} e^{n_{s-1}} \ldots a^{n_{2}} e^{n_{1}}\right), \quad h\left(a^{n_{4}} e^{n_{3}} a^{n_{2}} e^{n_{1}}\right), \quad \ldots, \quad h\left(a^{n_{2}} e^{n_{1}}\right) .
$$

This list, too, is unimodal (decreasing and then increasing). The suffix valleys are the decreasing terms at the right of the sequence, while the prefix valleys are the increasing terms at the left of the sequence. In case the sequence has a unique minimum, it is considered as both a suffix and prefix valley.

We now give the definition of peaks and valleys which holds also for words that are not run-unimodal. For a word $w=w_{n} \ldots w_{1}$ write $w_{[j}=w_{j} \ldots w_{1}$.

Suffix peaks and valleys:

- $y$ is a suffix peak if there exists a $j$ such that $h\left(w_{[j}\right)=y$, and either $y \geq h\left(w_{[\ell}\right)$ for all $\ell$, or there exists a $J>j$ such that for all $\ell<J, h\left(w_{[J}\right)<h\left(w_{[\ell}\right) \leq y$.

- $y$ is a suffix valley if there exists a $j$ such that $h\left(w_{[j}\right)=y$, and either $y \leq h\left(w_{[\ell}\right)$ for all $\ell$, or there exists a $J>j$ such that for all $\ell<J, h\left(w_{[J}\right)>h\left(w_{[\ell}\right) \geq y$.

Prefix peaks and valleys are defined likewise (by quantifying over $J<j$ rather than $J>j$ ):

- $y$ is a prefix peak if there exists a $j$ such that $h\left(w_{[j}\right)=y$, and either $y \geq h\left(w_{[\ell}\right)$ for all $\ell$, or there exists a $J<j$ such that for all $\ell>J, h\left(w_{[J}\right)<h\left(w_{[\ell}\right) \leq y$.

- $y$ is a prefix valley if there exists a $j$ such that $h\left(w_{[j}\right)=y$, and either $y \leq h\left(w_{[\ell}\right)$ for all $\ell$, or there exists a $J<j$ such that for all $\ell>J, h\left(w_{[J}\right)>h\left(w_{[\ell}\right) \geq y$.

A simple case analysis shows that the four lists are invariant under application of the identities in $\mathcal{G}$. The lists distinguish among run-unimodal words because they are the alternating sums of prefixes or suffixes of the sequence of exponents. 

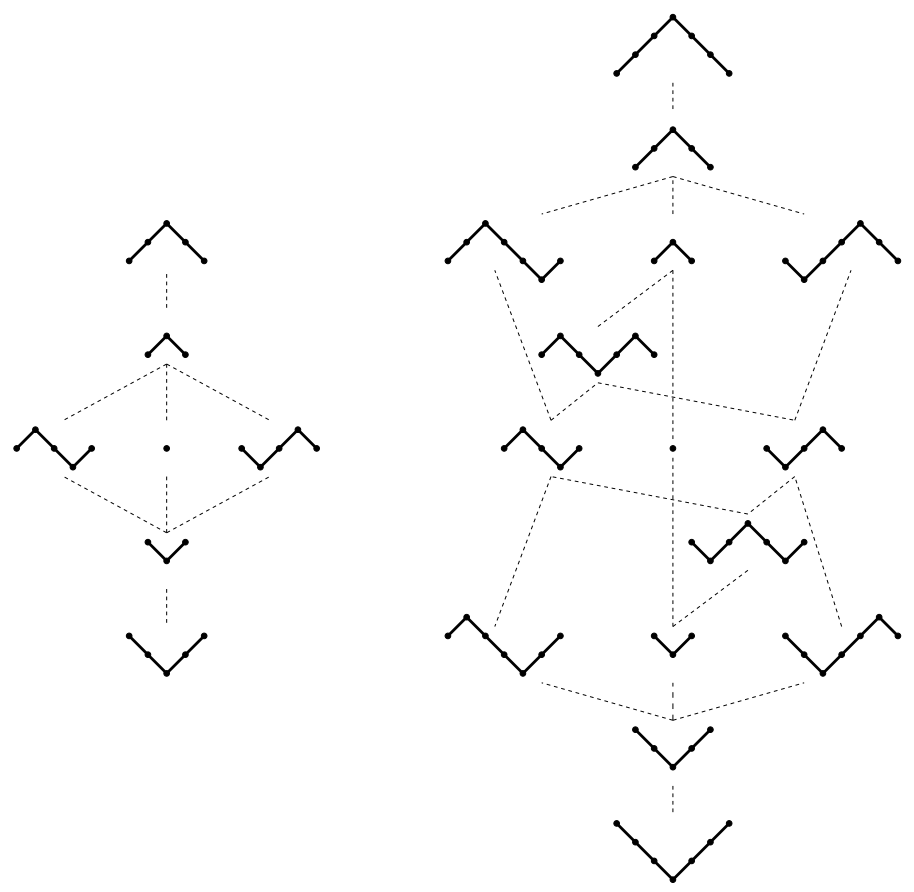

Figure 1: Hasse diagrams for fragments of $\mathfrak{S}$. Left: the words of length up to 4 . Right: the words of length up to 6 . Key: $a=/$ and $e=\backslash$.

\section{Proof of Theorem 7}

Outline of the proof:

(a) We introduce a second order, the layout order $\leq_{L}$, on $F^{0}$ (Definitions 9-11, Proposition 12), and show that after passing to the quotient $F^{0} / \mathcal{G}$ (justified in Lemma 13), no two elements of $F^{0} / \mathcal{G}$ are equivalent with respect to $\leq_{L}$ (Proposition 14).

The fact that $\leq_{L}$ is a refinement of $\leq$ on $F^{0} / \mathcal{G}$ will be implied by Corollary 4 ; the converse will take more work:

(b) In Propositions 15 and 16 we give concrete necessary and sufficient conditions for the relations $\leq_{L}$ and $\not_{L}$.

(c) In Proposition 17 we use the characterization from Proposition 16 to show that $\leq$ is a refinement of $\leq_{L}$, thereby obtaining their equivalence, and thus the equivalence of $F^{0} / \mathcal{G}$ and $\mathfrak{S}$.

\subsection{Words and their layouts}

Definition 9. Let $\bar{F}$ be the free semigroup of even-length words on $\left\{a, e, \varepsilon^{2}\right\}$. (I.e., the character $\varepsilon$ must occur in pairs; the length of each $\varepsilon$ is 1 .) Set $h(\varepsilon)=0$; and as before, $h(e)=1$ and $h(a)=-1$. 
Let $\bar{F}^{0}$ be the subsemigroup of $\bar{F}$ consisting of words of height 0 .

For $w=w_{n} \ldots w_{1}$ and $w^{\prime}=w_{m}^{\prime} \ldots w_{1}^{\prime}$ in $\bar{F}^{0}$, write $w \leq_{L} w^{\prime}$ if $n=m$ and if for every $j, h\left(w_{[j}\right) \leq h\left(w_{[j}^{\prime}\right)$.

$F$ is the quotient of $\bar{F}$ by the relation $\varepsilon^{2}=1$; we freely abuse notation by identifying $F$ with the quotient representatives lacking $\varepsilon$ 's. Height is preserved under this quotient map. See the diagram of semigroup mappings:

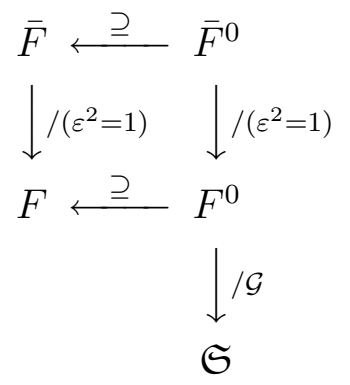

Let $\varepsilon^{2}$ act on each of $\{0,1\}^{U}$ and $\{0,1\}^{V}$ as the identity map. Then words in $\bar{F}$ map $\{0,1\}^{U}$ to itself. With this definition, the order $\leq$ on $F$ makes sense also on $\bar{F}$ :

Definition 10. If $\bar{w}$ and $\bar{w}^{\prime}$ are words in $\bar{F}$, write $\bar{w} \leq \bar{w}^{\prime}$ if $\bar{w} S \leq \bar{w}^{\prime} S$ for all $G$ and $S \in\{0,1\}^{U}$.

Plainly, $\bar{w} \leq \bar{w}^{\prime}$ in $\bar{F}$ if and only if $\bar{w} /\left(\varepsilon^{2}=1\right) \leq \bar{w}^{\prime} /\left(\varepsilon^{2}=1\right)$ in $F$.

The key connection between $\leq$ and $\leq_{L}$ is created by "lifting" from $F^{0}$ to $\bar{F}^{0}$ :

Definition 11. Let $w_{2}, w_{1} \in F^{0}$. Write $w_{2} \leq_{L} w_{1}$ if there exist $\bar{w}_{2}, \bar{w}_{1} \in \bar{F}^{0}$ such that $w_{2}=\bar{w}_{2} /\left(\varepsilon^{2}=1\right), w_{1}=\bar{w}_{1} /\left(\varepsilon^{2}=1\right)$, and $\bar{w}_{2} \leq_{L} \bar{w}_{1}$.

A word $\bar{w} \in \bar{F}^{0}$ such that $w=\bar{w} /\left(\varepsilon^{2}=1\right)$ will be termed a layout of $w$; Figure 2 motivates the terminology.

A characterization equivalent to Definition 11 is that (with $s_{1}$ and $s_{2}$ being the respective lengths of $w_{1}$ and $w_{2}$ ), there exist two sequences of indices $k$ and $k^{\prime}$ such that (with $w_{i, j} \in\{a, e\}$ for $i \in\{1,2\}$ being the characters of the two sequences):

1. $s_{2}=k^{\prime}\left(s_{1}\right) \geq k\left(s_{1}\right) \geq \ldots \geq k^{\prime}(0) \geq k(0)=0$

2. $k(j+1) \leq k^{\prime}(j)+1$ for all $j$

3. $k^{\prime}(j)-k(j)$ is even for all $j ; \max \{j: k(j)=\ell\}-\min \left\{j: k^{\prime}(j)=\ell\right\}$ is even for all $\ell$.

4. If $0 \leq j \leq s_{1}$ and $k(j) \leq K \leq k^{\prime}(j)$ then $h\left(w_{2, K} \ldots w_{2,1}\right) \leq h\left(w_{1, j} \ldots w_{1,1}\right)$.

These functions express how $w_{1}$ is laid out above $w_{2}$ : a nontrivial interval $k(j)<k^{\prime}(j)$ indicates an insertion of $\varepsilon^{2}$ 's in $w_{1}$ above the segment $w_{2, k^{\prime}(j)} \ldots w_{2, k(j)}$. A nontrivial interval $J=\min \left\{j: k^{\prime}(j)=\ell\right\}<\max \{j: k(j)=\ell\}=J^{\prime}$ indicates an insertion of $\varepsilon^{2}$ 's in $w_{2}$ below the segment $w_{1, J^{\prime}} \ldots w_{1, J}$.

The claim that $\leq_{L}$ is a partial order on $F^{0}$ requires justification:

Proposition 12. The relation $\leq_{L}$ on $F^{0}$ is transitive. 
Proof. Informally, transitivity holds because $\varepsilon^{2}$ 's may be inserted as needed. More carefully, let $w_{3} \leq_{L} w_{2}$ and $w_{2} \leq_{L} w_{1}$ in $F^{0}$, and write $w_{i}=w_{i, s_{i}} \ldots w_{i, 1}$. Then there exist sequences $k_{1}, k_{1}^{\prime}, k_{2}$ and $k_{2}^{\prime}$ such that

$$
\begin{aligned}
& s_{2}=k_{1}^{\prime}\left(s_{1}\right) \geq k_{1}\left(s_{1}\right) \geq \ldots \geq k_{1}^{\prime}(0) \geq k_{1}(0)=0 \\
& s_{3}=k_{2}^{\prime}\left(s_{2}\right) \geq k_{2}\left(s_{2}\right) \geq \ldots \geq k_{2}^{\prime}(0) \geq k_{2}(0)=0
\end{aligned}
$$

such that $k_{1}(j+1) \leq k_{1}^{\prime}(j)+1$ and $k_{2}(j+1) \leq k_{2}^{\prime}(j)+1$ for all $j$, and:

$$
\begin{aligned}
& \text { If } 0 \leq j \leq s_{1} \text { and } k_{1}(j) \leq K \leq k_{1}^{\prime}(j), \quad \text { then } \quad h\left(w_{2, K} \ldots w_{2,1}\right) \leq h\left(w_{1, j} \ldots w_{1,1}\right) \\
& \text { If } 0 \leq j \leq s_{2} \text { and } k_{2}(j) \leq K \leq k_{2}^{\prime}(j), \quad \text { then } \quad h\left(w_{3, K} \ldots w_{3,1}\right) \leq h\left(w_{2, j} \ldots w_{2,1}\right) .
\end{aligned}
$$

Composing, we find that $k_{2}\left(k_{1}(j+1)\right) \leq k_{2}\left(k_{1}(j)+1\right) \leq k_{2}\left(k_{1}(j)\right)+1$ and that:

$$
\text { If } 0 \leq j \leq s_{1} \text { and } k_{2}\left(k_{1}(j)\right) \leq K \leq k_{2}^{\prime}\left(k_{1}^{\prime}(j)\right) \text {, then } \quad h\left(w_{3, K} \ldots w_{3,1}\right) \leq h\left(w_{1, j} \ldots w_{1,1}\right) \text {. }
$$

The composition provides common layouts for the three words so that the height of the suffixes are in the order $h\left(\bar{w}_{1_{[j}}\right) \geq h\left(\bar{w}_{2_{[j}}\right) \geq h\left(\bar{w}_{3_{[j}}\right)$ for all $j$.

Lemma 13. For any $w, w^{\prime} \in F^{0}$ that are equivalent in $F^{0} / \mathcal{G}, w \leq_{L} w^{\prime} \leq_{L} w$.

Proof. It is enough to show that for any $w_{1}, w_{2}$ and $n, w_{1} e^{n} w_{2} \leq_{L} w_{1} e^{n} a^{n} e^{n} w_{2}$ and $w_{1} e^{n} a^{n} e^{n} w_{2} \leq_{L} w_{1} e^{n} w_{2}$. We describe insertions of $\varepsilon^{2}$ 's that demonstrate each inequality. For the first make the insertion $w_{1} e^{n} \varepsilon^{2 n} w_{2} \leq_{L} w_{1} e^{n} a^{n} e^{n} w_{2}$. For the second make the insertion $w_{1} e^{n} a^{n} e^{n} w_{2} \leq_{L} w_{1} \varepsilon^{2 n} e^{n} w_{2}$.

This lemma means that we can define $\leq_{L}$ on $F^{0} / \mathcal{G}$ (i.e., among equivalence classes of words) by using any representatives in $F^{0}$ of those classes.

Proposition 14. The relation $\leq_{L}$ on $F^{0} / \mathcal{G}$ is transitive and acyclic (i.e., it is a partial order).

Proof. Transitivity follows from Proposition 12.

In order to show acyclicity it suffices, because of Lemmas 8 and 13, to consider rununimodal words $w, w^{\prime} \in F^{0}$, and to show that $w \leq_{L} w^{\prime} \leq_{L} w$ implies $w=w^{\prime}$.

Let the norm of a run-unimodal word $a^{n_{s}} e^{n_{s-1}} \ldots a^{n_{2}} e^{n_{1}}$ be $N\left(a^{n_{s}} e^{n_{s-1}} \ldots a^{n_{2}} e^{n_{1}}\right)=$ $\max _{j}\left|\sum_{i=1}^{j}(-1)^{i+1} n_{i}\right|$. The norm is achieved at no more than one peak and at no more than one valley. Without loss of generality suppose $N(w)$ is achieved at a peak. Then $w^{\prime}$ must share the same norm and also achieve it at a peak. Fix layouts $\bar{w}_{1}, \bar{w}_{2} \in \bar{F}^{0}$ of $w$, and a layout $\bar{w}^{\prime} \in \bar{F}^{0}$ of $w^{\prime}$, such that for all $j, \bar{w}_{2_{[j}} \leq \bar{w}_{[j}^{\prime} \leq \bar{w}_{1_{[j}}$. Let $w=$ $a^{n_{s}} e^{n_{s-1}} \ldots a^{n_{2}} e^{n_{1}}$ and $w^{\prime}=a^{m_{r}} e^{n_{r-1}} \ldots a^{m_{2}} e^{m_{1}}$. Define $T_{1}(j)$ to be the smallest $t$ for which $h\left(\bar{w}_{1_{[t}}\right)=\sum_{i=1}^{j}(-1)^{i+1} n_{i}$; define $T_{2}(j)$ to be the smallest $t$ for which $h\left(\bar{w}_{2_{[t}}\right)=$ $\sum_{i=1}^{j}(-1)^{i+1} n_{i}$; and define $T^{\prime}(j)$ to be the smallest $t$ for which $h\left(\bar{w}_{[t}^{\prime}\right)=\sum_{i=1}^{j}(-1)^{i+1} m_{i}$. Write $T_{1}(0)=T_{2}(0)=T^{\prime}(0)=0$.

Let the norms of $w$ and $w^{\prime}$ be achieved at the suffixes $e^{n_{k}} \ldots e^{n_{1}}$ and $e^{m_{\ell}} \ldots e^{m_{1}}$. We argue that $k=\ell$ and that for $i<k, n_{i}=m_{i}$; a similar argument will show that $n-k=m-\ell$ and that for $i<k, n_{i}=m_{i}$. To begin with, we can easily identify 
where the norms of the two words are achieved: there must be an $r$ such that $N(w)=$ $h\left(\bar{w}_{2_{[r}}\right)=h\left(\bar{w}_{[r}^{\prime}\right)=h\left(\bar{w}_{1_{[r}}\right)$. Next we claim that for $0 \leq j<k$, and for $t_{j}$ defined by $t_{j}=\max \left\{T_{1}(j), T_{2}(j)\right\}$, we have $\sum_{i=1}^{j}(-1)^{i+1} n_{i}=h\left(\bar{w}_{2_{\left[t_{j}\right.}}\right)=h\left(\bar{w}_{\left[t_{j}\right.}^{\prime}\right)=h\left(\bar{w}_{1_{\left[t_{j}\right.}}\right)$. This holds trivially for $j=0$; if it fails, let $j$ be the first counterexample. Suppose $j$ is odd (the even case follows by a similar argument). Observe that for $t_{j-1} \leq q \leq T_{2}(j)$, $\sum_{i=1}^{j-1}(-1)^{i+1} n_{i}=h\left(\bar{w}_{2_{\left[t_{j-1}\right.}}\right) \leq h\left(\bar{w}_{2_{[q}}\right) \leq h\left(\bar{w}_{2_{\left[T_{2}(j)\right.}}\right)=\sum_{i=1}^{j}(-1)^{i+1} n_{i}$. So $T_{1}(j) \leq T_{2}(j)<$ $T_{1}(j+1)$. But for $j$ to be a counterexample, i.e., for $h\left(\bar{w}_{\left.1^{1 t_{j}}\right)}\right)$ to be strictly larger than $h\left(\bar{w}_{\left.2_{\left[t_{j}\right.}\right)}\right)$ it is necessary that $T_{1}(j+1)<T_{2}(j)$. Contradiction.

We now see that in each of the intervals $t_{j} \leq q \leq t_{j+1}$ (for $0 \leq j<k$ ), the height of $\bar{w}^{\prime}$ is bounded by $\sum_{i=1}^{j}(-1)^{i+1} n_{i} \leq h\left(\bar{w}_{[q}^{\prime}\right) \leq \sum_{i=1}^{j+1}(-1)^{i+1} n_{i}$, and that the first of these inequalities is saturated at $t_{j}$ while the second is saturated at $t_{j+1}$ (with the understanding $t_{k}=r$ ). Since $w^{\prime}$ is run-unimodal (recall that this means that there are no occurrences in it of $e^{n} a^{n} e^{n}$ or $a^{n} e^{n} a^{n}$ for $n \geq 1$ ), it follows that $w^{\prime}=w$.

\subsection{Characterizations of the layout order}

We have already discussed the paths corresponding to words but we now need to be more systematic. The minimal layout of the empty word is the point $(0,0)$ in the plane; if the leftmost vertex in the layout of a word $w$ is $(x, y)$, then the minimal layout of aw augments the minimal layout of $w$ with a diagonal from $(x, y)$ to a new vertex at $(x-1, y-1)$; similarly, the minimal layout of ew augments the minimal layout of $w$ with a diagonal from $(x, y)$ to a new vertex at $(x-1, y+1)$. It is these minimal layouts which are depicted in Figure 1. A layout of a word is any path obtained from its minimal layout by insertions of even-length horizontal segments, representing insertions of $\varepsilon^{2}$ 's. The pictorial interpretation of the layout order, which we henceforth discuss only for rununimodal words, is: $w \geq_{L} w^{\prime}$ if and only if $w$ and $w^{\prime}$ have layouts ending at a common point, so that the $w$ path is nowhere strictly beneath the $w^{\prime}$ path.

We show in the next section that $\geq_{L}$ and $\geq$ impose the same order on run-unimodal words. First, though, it will be useful to give another, "constructive," characterization of the order.

Let $w=a^{n_{s}} e^{n_{s-1}} \ldots a^{n_{2}} e^{n_{1}}$; define the lower partial heights $h_{0}(w), \ldots, h_{s / 2}(w)$ by $h_{i}(w)=\sum_{j=1}^{i} n_{2 j-1}-n_{2 j}$. (Observe that $h_{0}(w)=h_{s / 2}(w)=0$.) Similarly define the upper partial heights $H_{1}(w), \ldots, H_{s / 2}(w)$ by $H_{i}(w)=h_{i}(w)+n_{2 i}$. If a layout of $w$ does not "stretch out" any of its minima with horizontal segments, let $x_{0}(w), \ldots, x_{s / 2}(w)$ denote the horizontal coordinates of its minima, thus $\left(x_{0}(w), h_{0}(w)\right), \ldots,\left(x_{s / 2}(w), h_{s / 2}(w)\right)$ are the local minima of the layout.

Let $w^{\prime}=a^{n_{s^{\prime}}^{\prime}} e^{n_{s^{\prime}}^{\prime}-1} \ldots a^{n_{2}^{\prime}} e^{n_{1}^{\prime}}$. If a layout of $w^{\prime}$ does not "stretch out" any of its maxima with horizontal segments, let $X_{1}\left(w^{\prime}\right), \ldots, X_{s^{\prime} / 2}\left(w^{\prime}\right)$ denote the horizontal coordinates of its maxima, thus $\left(X_{1}\left(w^{\prime}\right), H_{1}\left(w^{\prime}\right)\right), \ldots,\left(X_{s^{\prime} / 2}\left(w^{\prime}\right), H_{s^{\prime} / 2}\left(w^{\prime}\right)\right)$ are the local maxima of the layout.

Naturally, in any layout of $w$ and $w^{\prime}, x_{s / 2}(w)<\ldots<x_{0}(w)$ and $X_{s^{\prime} / 2}\left(w^{\prime}\right)<\ldots<$ 
$X_{1}\left(w^{\prime}\right)$. Now define the following functions:

$$
\begin{aligned}
& I_{0}(j)=\min \left\{i: H_{i}(w) \geq H_{j}\left(w^{\prime}\right)\right\} \quad(\text { or }=s / 2 \text { if the set is empty }) \\
& I_{1}(j)=\max \left\{i: H_{i}(w) \geq H_{j}\left(w^{\prime}\right)\right\} \quad(\text { or }=0 \text { if the set is empty }) \\
& J_{0}(i)=\min \left\{j: h_{j}\left(w^{\prime}\right) \leq h_{i}(w)\right\} \quad\left(\text { or }=s^{\prime} / 2\right. \text { if the set is empty) } \\
& J_{1}(i)=\max \left\{j: h_{j}\left(w^{\prime}\right) \leq h_{i}(w)\right\} \quad(\text { or }=0 \text { if the set is empty) }
\end{aligned}
$$

Proposition 15. $w \geq_{L} w^{\prime}$ if and only if the inequalities $x_{s / 2}(w)<\ldots<x_{0}(w)$ and $X_{s^{\prime} / 2}\left(w^{\prime}\right)<\ldots<X_{1}\left(w^{\prime}\right)$ are consistent with the following inequalities (in which we range over all $1 \leq j \leq s^{\prime} / 2$ and $\left.1 \leq i \leq s / 2-1\right)$ :

1. $x_{I_{1}(j)}(w)<X_{j}\left(w^{\prime}\right)<x_{I_{0}(j)-1}(w)$.

2. $X_{J_{1}(i)+1}\left(w^{\prime}\right)<x_{i}(w)<X_{J_{0}(i)}\left(w^{\prime}\right)$.

The meaning of these inequalities is this: the first set demands that the peaks of $w^{\prime}$ fit beneath sufficiently high peaks of $w$. The second set demands that the valleys of $w$ fit above sufficiently deep valleys of $w^{\prime}$. See Figures 2, 3 for examples.

Proof. If the inequalities are consistent, then any extension to a linear order specifies for each valley of $w$ the two peaks of $w^{\prime}$ it fits between; and for each peak of $w^{\prime}$, the two valleys of $w$ it fits between. The inequalities ensure that in this configuration the $w^{\prime}$ path may always remain beneath the $w$ path. (This may require introducing some plateaus in the peaks of $w$ or the valleys of $w^{\prime}$.)

Conversely, if $w \geq_{L} w^{\prime}$, then the layout provides horizontal coordinates $x_{s / 2}(w)<$ $\ldots<x_{0}(w)$ and $X_{s^{\prime} / 2}\left(w^{\prime}\right)<\ldots<X_{1}\left(w^{\prime}\right)$; there are no duplications between these lists (except possibly at the ends) since the height of $w^{\prime}$ at any $X_{j}\left(w^{\prime}\right)$ (other than possibly at $\left.j \in\left\{0, s^{\prime} / 2\right\}\right)$ is positive, and the height of $w$ at any $x_{i}(w)$ (other than at $i \in\{0, s / 2\}$ ) is negative. The inequalities of types 1,2 are then implied by the assumption that the layout of $w^{\prime}$ is beneath the layout of $w$.

Proposition 15 provides a "witness," or positive characterization, for the relation $\geq_{L}$; it will be useful to also have a witness for the relation $\Varangle_{L}$. Let $k(w)$ be the set of one or two indices $i$ at which $h_{i}(w)$ is minimal; let $K\left(w^{\prime}\right)$ be the set of one or two indices $j$ at which $H_{j}\left(w^{\prime}\right)$ is maximal.

Proposition 16. $w \Varangle_{L} w^{\prime}$ if and only if at least one of the following conditions holds:

1. $\max H_{j}\left(w^{\prime}\right)>\max H_{i}(w)$.

2. $\min h_{j}\left(w^{\prime}\right)>\min h_{i}(w)$.

3. For some $i \leq \min k(w)$ and $j \leq \min K\left(w^{\prime}\right), H_{j}\left(w^{\prime}\right)>H_{i}(w)$ and $h_{i}(w)<h_{j-1}\left(w^{\prime}\right)$.

4. For some $i \geq \max k(w)$ and $j \geq \max K\left(w^{\prime}\right), H_{i+1}(w)<H_{j}\left(w^{\prime}\right)$ and $h_{j}\left(w^{\prime}\right)>h_{i}(w)$.

Condition 3 describes the possible obstructions to $w \geq_{L} w^{\prime}$ within the prefixes; condition 4 describes the corresponding possible obstructions to $w \geq_{L} w^{\prime}$ within the suffixes. 
Proof. We start with the "if." Clearly either condition 1 or 2 imply $w \Varangle_{L} w^{\prime}$. Next assume condition 3 (the argument for condition 4 is similar). Then there exist $i \leq \min k(w)$ and $j \leq \min K\left(w^{\prime}\right)$ for which $H_{j}\left(w^{\prime}\right)>H_{i}(w)$ and $h_{i}(w)<h_{j-1}\left(w^{\prime}\right)$; we observe the following two constraints on any pair of layouts that maintain $w$ above $w^{\prime}$. Since $h_{i}(w)<h_{j-1}\left(w^{\prime}\right)$, the $i^{\prime}$ th valley of $w$ cannot be above the $j-1^{\prime}$ th valley of $w^{\prime}$, or anything to its right; so it must be laid out strictly to the left of the $j^{\prime}$ th peak of $w^{\prime}$. Similarly, since $H_{j}\left(w^{\prime}\right)>H_{i}(w)$, the $j$ 'th peak of $w^{\prime}$ cannot be below the $i$ 'th peak of $w$, or anything to its right; so it must be laid out strictly to the left of the $i$ 'th valley of $w$. These constraints are contradictory.

The "only if" is constructive. A visual depiction is useful. Form a two-dimensional array of vertices with coordinates $(j, i)$ where $j$ ranges (from right to left) from 0 to $s^{\prime} / 2$, and $i$ ranges (from top to bottom) from 1 to $s / 2$. The upper-right vertex, with coordinates $(0,1)$, is denoted $A$; the lower-right vertex, with coordinates $\left(s^{\prime} / 2, s / 2\right)$, is denoted $D$. Place horizontal and vertical edges among these vertices as follows:

Vertical edges: vertices $(j, i)$ and $(j, i-1)$ are connected if $J_{0}(i) \leq j \leq J_{1}(i)$.

Horizontal edges: vertices $(j, i)$ and $(j-1, i)$ are connected if $I_{0}(j) \leq i \leq I_{1}(j)$.

A layout in which $w$ dominates $w^{\prime}$ corresponds (according to the tightened version of Proposition 15$)$ to a monotone path between $A$ and $D$. Use of a vertical edge $((j, i),(j, i-$ $1)$ ) in this path corresponds to interleaving $x_{i}$ between $X_{j}$ and $X_{j+1}$. Use of a horizontal edge $((j, i),(j-1, i))$ corresponds to interleaving $X_{j}$ between $x_{i}$ and $x_{i-1}$.

To show the "only if," suppose there is no monotone path between $A$ and $D$. If either condition 1 or 2 are fulfilled, we are done. (Condition 1 asserts that for some $j$, the interval $\left[I_{0}(j), I_{1}(j)\right]$ is empty; equivalently, there are no horizontal edges across the $i$ th column of the grid. Similarly, condition 2 asserts that for some $i$, the interval $\left[J_{0}(i), J_{1}(i)\right]$ is empty; equivalently, there are no vertical edges across some row of the grid.) If neither condition 1 nor condition 2 are fulfilled, we need to show that either condition 3 or 4 is. Failure of 1 and 2 implies that there are some adjacent columns, including at least the columns corresponding to the set $K\left(w^{\prime}\right)$, in which the vertical edges go all the way from top to bottom; and that there are some adjacent rows, including at least the rows corresponding to $k(w)$, in which the horizontal edges go all the way from left to right. These rows and columns intersect; we let $C=\left(c_{1}, c_{2}\right)$ denote the lower-left vertex in the intersection, and $B=\left(b_{1}, b_{2}\right)$ the upper-right vertex in the intersection. Clearly, if there were a monotone path between $A$ and $D$, there would be one which passes through $C$ and $B$. Hence there is either no monotone path between $D$ and $C$, or no monotone path between $A$ and $B$. Without loss of generality we suppose the latter, and show this implies condition 3 ; in similar fashion the former implies condition 4.

Condition 3 is implied by the statement that: $\left({ }^{*}\right)$ For some $a_{1} \geq j>0$ and $a_{2} \geq i>1$, there is no horizontal edge $((j, i-1),(j-1, i-1))$ and no vertical edge $((j-1, i),(j-$ $1, i-1))$.

To prove $(*)$, consider the set of vertices $(j, i)$ for which $j \leq b_{1}, i \leq b_{2}$, there is a monotone path from $\left(b_{1}, b_{2}\right)$ to $(j, i)$, and $(j, i)$ is connected by straight lines (possibly of length 0$)$ to the top and right boundaries (in other words all of the edges $\left(\left(j^{\prime}, i\right),\left(j^{\prime}-\right.\right.$ $1, i))_{j^{\prime} \leq j} \bigcup\left(\left(j, i^{\prime}\right),\left(j, i^{\prime}-1\right)\right)_{i^{\prime} \leq i}$ are in the graph). $B$ itself belongs to this set, and the set cannot contain $A$. It also cannot contain any vertex on the right or top edge of the grid 
since that vertex would then be connected by monotone paths both to $B$ and to $A$. So there are $j>0$ and $i>1$ such that $(j, i)$ in the set but neither $(j, i-1)$ nor $(j-1, i)$ is in the set. Since $(j, i-1)$ is connected by a straight line to the top boundary, it must not be connected by a straight line to the right boundary; this necessarily means that already the first edge on that line, $((j, i-1),(j-1, i-1))$, is missing. Similarly, since $(j-1, i)$ is connected by a straight line to the right boundary, it must not be connected by a straight line to the top boundary; this necessarily means that already the first edge on that line, $((j-1, i),(j-1, i-1))$, is missing.
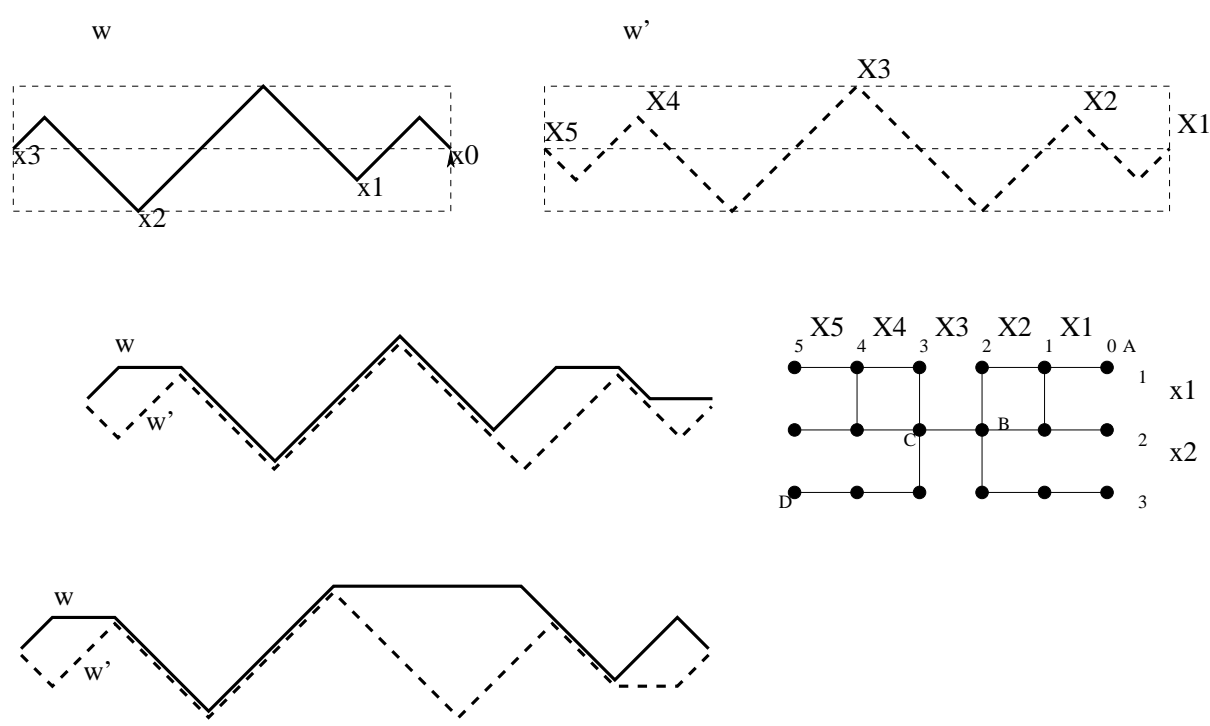

Figure 2: An example in which $w \geq w^{\prime}: w=a e^{3} a^{4} e^{3} a^{2} e, w^{\prime}=e a^{2} e^{3} a^{4} e^{4} a^{3} e^{2} a$. Left, middle and bottom: two layouts showing domination. Right, middle: the graph of constraints.

\subsection{Equivalence of $\leq$ and $\leq_{L}$}

The following Proposition completes the characterization of the partial order on $F^{0} / \mathcal{G}$.

Proposition 17. $w \geq w^{\prime}$ if and only if their equivalent run-unimodal words $\hat{w}$ and $\hat{w}^{\prime}$ satisfy $\hat{w} \geq_{L} \hat{w}^{\prime}$.

In particular, distinct run-unimodal words are inequivalent in $\mathfrak{S}$, which completes the proof of Theorem 7 .

Proof. For simplicity of notation we suppose that $w$ and $w^{\prime}$ are themselves run-unimodal.

Sufficiency $\left(w \geq_{L} w^{\prime} \Longrightarrow w \geq w^{\prime}\right)$ follows from Corollary 4 .

Necessity $\left(w \geq w^{\prime} \Longrightarrow w \geq_{L} w^{\prime}\right)$ follows from examining certain test instances. We use the characterization of $\geq_{L}$ given in Proposition 16. 

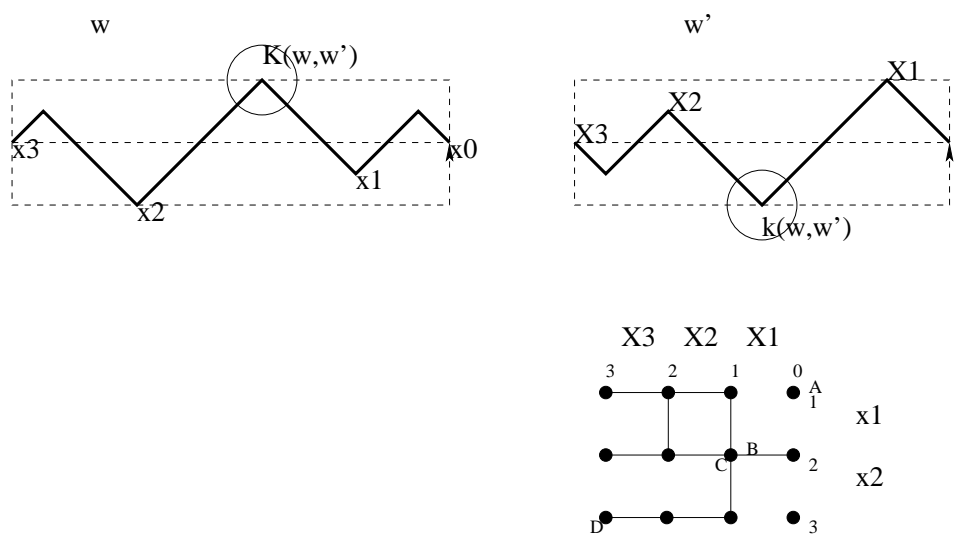

Figure 3: An example in which $w \nsupseteq w^{\prime}: w=a e^{3} a^{4} e^{3} a^{2} e, w^{\prime}=e a^{2} e^{3} a^{4} e^{2}$. The pair $\left\{x_{1}, X_{1}\right\}$ are an obstruction to domination.

There are two general classes of test instances: the first class is used to determine the increasing portion of the unimodal sequence, $n_{r}>\ldots>n_{1} \geq 0$, and the second class is used to determine the decreasing portion, $0 \leq n_{s}<n_{s-1}<\ldots<n_{r+1}$.

In the first class of instances the graphs, called $G_{K}^{0}$ or $G_{K}^{1}$, are chains of $K$ vertices, as in Figure 4; in $G_{K}^{1}, U$ consists of the odd-indexed vertices and includes the leftmost vertex of the chain, while in $G_{K}^{0}, U$ consists of the even-indexed vertices, and $V$ includes the left-most vertex of the chain. In any $G_{K}^{\beta}(\beta \in\{0,1\}),|U|=\lfloor(K+\beta) / 2\rfloor$. Let $S_{L}$ $(0 \leq L \leq|U|)$ denote the $L$ leftmost vertices of $U$, and $T_{L}(0 \leq L \leq|V|)$ denote the $L$ leftmost vertices of $V$.

Now, and later, we have occasion to apply the operators $a$ and $e$ in situations in which the ambient graph $G$ is not clear from context: in such cases we write "eS $[G]$ " or " $a S[G]$," or we write "[G]" at the end of an equation to indicate it applies to all operators within.

Observe that:

1. If $0<L<|U|$ then $a S_{L}=T_{L-\beta}\left[G_{K}^{\beta}\right]$ and $e S_{L}=T_{L+1-\beta}\left[G_{K}^{\beta}\right]$.

2. If $L=0$ then $a S_{L}=e S_{L}=\emptyset\left[G_{K}^{\beta}\right]$.

3. If $L=|U|$ then $a S_{L}=e S_{L}=V\left[G_{K}^{\beta}\right]$.

Similarly,

1. If $0<L<|V|$ then $a T_{L}=S_{L-1+\beta}\left[G_{K}^{\beta}\right]$ and $e T_{L}=S_{L+\beta}\left[G_{K}^{\beta}\right]$.

2. If $L=0$ then $a T_{L}=e T_{L}=\emptyset\left[G_{K}^{\beta}\right]$.

3. If $L=|V|$ then $a T_{L}=e T_{L}=U\left[G_{K}^{\beta}\right]$.

Putting these observations together, we see that if a sequence of $e$ 's and $a$ 's are applied to a set $S_{L}$, the $e$ 's and $a$ 's act as inverses (the $e$ 's expanding the set, the $a$ 's contracting 


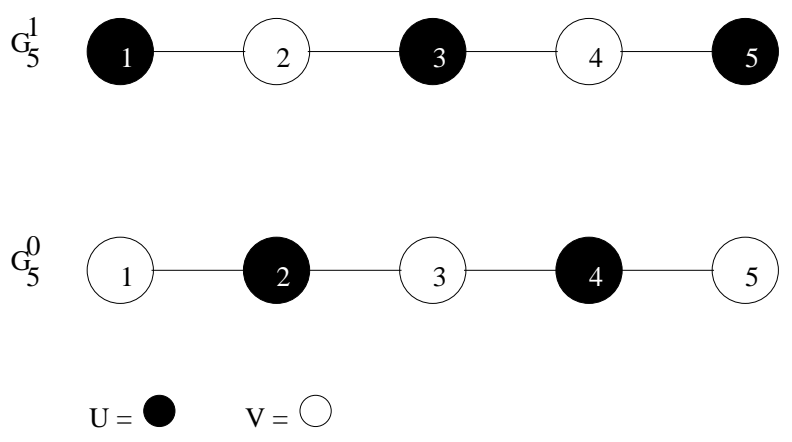

Figure 4: $G_{5}^{1}$ (above) and $G_{5}^{0}$ (below).

it) unless at any stage the set either becomes empty or expands to fill a whole part of the bipartite graph; either condition is irreversible.

We therefore conclude that for any word $w$ of height 0 and any $0<L<|U|$,

1. $w S_{L}=\emptyset\left[G_{K}^{\beta}\right]$ if there exists a prefix of $w$ (the $w_{1}$ in $w=w_{2} w_{1}$ ) of height $-2 L+\beta$ and if the shortest such prefix does not contain a prefix of height $K+\beta-2 L$.

2. $w S_{L}=U\left[G_{K}^{\beta}\right]$ if there exists a prefix of $w$ of height $K+\beta-2 L$ and if the shortest such prefix does not contain a prefix of height $-2 L+\beta$.

3. $w S_{L}=S_{L}\left[G_{K}^{\beta}\right]$ in all other cases.

These conclusions allow us to handle the first three of the conditions in Proposition 16. For condition (1), if we choose $K, \beta, L$ so that

$$
K+\beta-2 L=\max H_{i}\left(w^{\prime}\right) \quad \text { and } \quad-2 L+\beta=-1+\min \left\{\min h_{i}(w), \min h_{i}\left(w^{\prime}\right)\right\}
$$

(which can always be done), then $w^{\prime} S_{L}=U\left[G_{K}^{\beta}\right]$ and $w S_{L}=S_{L}\left[G_{K}^{\beta}\right]$. So $w^{\prime} S_{L} \nsubseteq$ $w S_{L}\left[G_{K}^{\beta}\right]$.

For condition (2), if we choose $K, \beta, L$ so that

$$
K+\beta-2 L=1+\max \left\{\max H_{i}\left(w^{\prime}\right), \max H_{i}(w)\right\} \quad \text { and }-2 L+\beta=\min h_{i}(w)
$$

(which can always be done), then $w^{\prime} S_{L}=S_{L}\left[G_{K}^{\beta}\right]$ and $w S_{L}=\emptyset\left[G_{K}^{\beta}\right]$. So $w^{\prime} S_{L} \nsubseteq$ $w S_{L}\left[G_{K}^{\beta}\right]$.

For condition (3) (with $i$ and $j$ as specified in that condition), if we choose $K, \beta, L$ so that

$$
K+\beta-2 L=H_{j}\left(w^{\prime}\right) \text { and }-2 L+\beta=h_{i}(w)
$$

(which can always be done), then $w^{\prime} S_{L}=U$ and $w S_{L}=\emptyset$. So $w^{\prime} S_{L} \not \subset w S_{L}\left[G_{K}^{\beta}\right]$.

In order to handle condition (4) we consider our second class of test instances. These graphs, called $H_{K, L, M, N}$, consist of: (a) A main stem, which is a chain of vertices labeled $A_{-N}^{0}, \ldots, A_{0}^{0}, \ldots, A_{K}^{0}$. (b) A branch, which is a chain of vertices labeled $A_{M+1}^{1}, \ldots, A_{L}^{1}$, connected to the main stem by an edge between $A_{M}^{0}$ and $A_{M+1}^{1}$. Observe that the last 
vertex of $U$ on the main stem is $A_{2\lfloor K / 2\rfloor}^{0}$. (We require $K, L, N \geq 0 ; K \geq M \geq-N$.) See Figure 5. In the bipartition of this graph, $U$ is the set of vertices with even subscripts, and $V$ the set with odd subscripts. The set $S$ consists of the vertices of $U$ with non-positive subscripts.

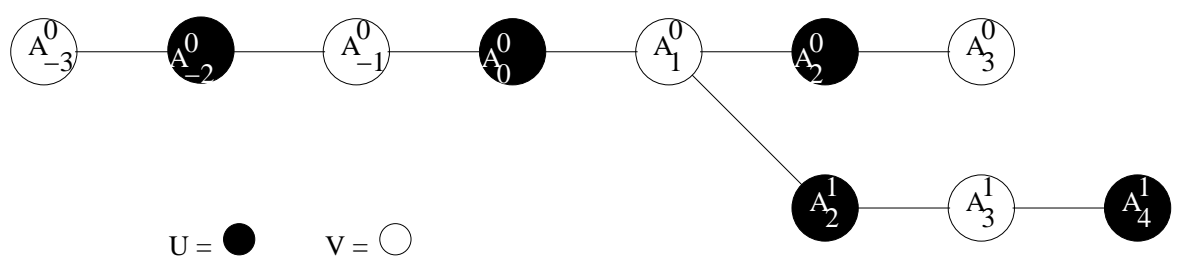

Figure 5: $H_{3,4,1,3}$

The essential property of the construction is this: Suppose that $L>\max H(w)$ and $-N<\min h(w)$. Then if $A_{2\lfloor K / 2\rfloor}^{0} \notin w S$ and if $w$ has a prefix $w_{1}$ such that $A_{2\lfloor K / 2\rfloor}^{0} \in w_{1} S$, then $w$ has a longer prefix $w_{2} w_{1}$ whose height is $2 M-K-1$.

From this we derive:

1. If $\max H_{i}(w)<2\lfloor K / 2\rfloor$ then $w S=S\left[H_{K, L, M, N}\right]$.

2. Suppose $\max H_{i}(w) \geq 2\lfloor K / 2\rfloor$. Let $w_{1}$ be the longest prefix of $w$ of height $2\lfloor K / 2\rfloor$. Then if $w$ has a prefix $w_{2} w_{1}$ of height $2 M-K-1, A_{2\lfloor K / 2\rfloor}^{0} \notin w S\left[H_{K, L, M, N}\right]$; otherwise, $A_{2\lfloor K / 2\rfloor}^{0} \in w S\left[H_{K, L, M, N}\right]$.

Now let $i$ and $j$ be as in condition (4). Set $M$ and $K$ so that

$$
2 M-K-1=h_{j}\left(w^{\prime}\right)-1 \quad \text { and } \quad\lfloor K / 2\rfloor=\left\lfloor H_{j}\left(w^{\prime}\right) / 2\right\rfloor .
$$

(This can always be done.) Also set

$$
L=1+\max \left\{\max H_{i}(w), H_{j}\left(w^{\prime}\right)\right\} \quad \text { and } \quad N=1-\min \left\{\min h_{i}(w), \min h_{j}\left(w^{\prime}\right)\right\} .
$$

(Larger values of $L$ and $N$ would also do.) Then $A_{2\lfloor K / 2\rfloor}^{0} \in w^{\prime} S\left[H_{K, L, M, N}\right]$, but $A_{2\lfloor K / 2\rfloor}^{0} \notin$ $w S\left[H_{K, L, M, N}\right]$. So $w^{\prime} S \nsubseteq w S\left[H_{K, L, M, N}\right]$.

\section{Discussion}

\subsection{General lattices}

The operations $a$ and $e$ make sense in somewhat greater generality. Let $\Gamma$ be any lattice, with meet $\wedge$ and join $\vee$. Define $e$ and $a$ by

$$
\begin{array}{ll}
e: \Gamma^{U} \rightarrow \Gamma^{V} & a: \Gamma^{U} \rightarrow \Gamma^{V} \\
(e(S))(v)=\bigvee_{u \in G(*, v)} S(u) & (a(S))(v)=\bigwedge_{u \in G(*, v)} S(u) \\
e: \Gamma^{V} \rightarrow \Gamma^{U} & a: \Gamma^{V} \rightarrow \Gamma^{U} \\
(e(T))(u)=\bigvee_{v \in G(u, *)} T(v) & (a(T))(u)=\bigwedge_{v \in G(u, *)} T(v)
\end{array}
$$


Instead of Definition 1 we can define $\dot{\leq}$ by

Definition 18. The relation $\dot{\leq}$ is defined on $F$ by: $w \leq w^{\prime}$ if $w S \leq w^{\prime} S$ for all $G, \Gamma$ and $S \in \Gamma^{U}$.

If the definition of $\mathfrak{S}$ is revised, again using Definition 2 but on the basis of $\dot{\leq}$, then Lemma 3 and Corollary 4 (as well as Corollary 5) go through without change. It follows that the newly-defined $\mathfrak{S}$ is again a quotient of $F^{0} / \mathcal{G}$. But then, considering $\{0,1\}$ as a special case of $\Gamma$, we see that Theorem 7 goes through without change. Because of this equivalence we have stated our main results in the more concrete setting of the Boolean algebra.

\subsection{Transposition}

Define transposition of words by $a^{T}=e, e^{T}=a$, and $\left(w_{1} w_{2}\right)^{T}=w_{2}^{T} w_{1}^{T}$. The semigroup $\mathfrak{S}$ is symmetric with respect to transposition: $w \geq w^{\prime}$ if and only if $w^{T} \geq w^{T}$. Is there a simple explanation for this that does not rely on the equivalence with the layout order?

\subsection{Enumeration}

How quickly does $\mathfrak{S}$ grow? That is, how many run-unimodal words are there of length $2 n$, and does this sequence have a closed-form generating function? Here are the data for $n \leq 6$. The " \pm " in all but the first row accounts for the fact that words may start with either $a$ or $e$.

\begin{tabular}{r|l|r}
\hline $2 n$ & the run-unimodal words of length $2 n$ (given by exponent sequence) & \# words \\
\hline 0 & 0 & 1 \\
2 & \pm 11 & 2 \\
4 & $\pm 22,121$ & 4 \\
6 & $\pm 33,231,132,1221$ & 8 \\
8 & $\pm 44,143,341,1331,2321,1232$ & 12 \\
10 & $\pm 55,154,451,1441,2332,2431,1342,1243,3421$ & 38 \\
12 & $\pm 66,165,561,1551,1254,4521,2541,1452,1353,3531,2442$, & \\
& $14421,12241,13431,2343,3432,24321,12342,123321$ & \\
\hline
\end{tabular}

At the time of writing, neither the sequence in the right-hand column nor its variant $(1,2,4,6,9,19)$ (which eliminates the factor of 2 for \pm , dropping the first term), was listed in the On-Line Encyclopedia of Integer Sequences [7].

Some sets of words related to those we study are well known. Words in $\bar{F}^{0}$ with the property that every prefix has nonnegative height are known as Schröder words or paths [12]; they appear in numerous guises [15], and see [14] for more on their enumeration and its history. Likewise, words in $F^{0}$ with the property that every prefix has nonnegative height are known as Dyck words or paths; they too appear in various contexts [15], while their enumeration is simpler, as they are counted by the Catalan numbers. 


\section{Acknowledgments}

Thanks to an anonymous referee for helpful comments on the paper.

\section{References}

[1] G. Birkhoff. Lattice Theory. Amer. Math. Soc., 3rd edition, 1967.

[2] C. J. Everett. Closure operations and Galois theory in lattices. Trans. Amer. Math. Soc., 55:514-525, 1944.

[3] G. Frobenius. Über zerlegbare determinanten. Sitzungsbericht der Königlich Preussischen Akademie Wissenschaften, XVIII:274-277, 1917.

[4] P. Hall. On representation of subsets. J. London Math. Soc., 10:26-30, 1935.

[5] D. König. Graphs and matrices. Mat. és Fiz. Lapok, 38:116-119, 1931.

[6] K. Menger. Zur allgemeinen kurventheorie. Fund. Math., 10:96-115, 1927.

[7] The on-line encyclopedia of integer sequences. http://oeis.org. Accessed June 22, 2010.

[8] O. Ore. Galois connexions. Trans. Amer. Math. Soc., 55:493-513, 1944.

[9] G. Pickert. Bemerkungen uber Galois-Verbindungen. Archiv. Math., 3:385-389, 1952.

[10] G. C. Rota. On the foundations of combinatorial theory. I. Theory of Möbius functions. Zeits. f. Wahrscheinlichkeitstheorie, 2:340-368, 1964.

[11] J. Schmidt. Beitrage für filtertheorie II. Math. Nachr., 10:197-232, 1953.

[12] E. Schröder. Vier kombinatorische probleme. Z. Math. Phys., 15:361-376, 1870.

[13] Z. Shmuely. The structure of Galois connections. Pacific J. Math., 54(2):209-225, 1974.

[14] R. P. Stanley. Hipparchus, Plutarch, Schröder, and Hough. American Mathematical Monthly, 104(4):344-350, 1997.

[15] R. P. Stanley. Enumerative Combinatorics. Cambridge U. Press, 1999. 